\title{
A short communication to define the overcurrent protection system of the CIGRE European benchmark distribution networks for RES penetration studies
}

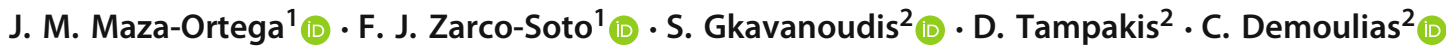

Received: 26 January 2021 / Accepted: 21 August 2021 / Published online: 15 September 2021

(c) The Author(s) 2021

\begin{abstract}
The use of clean energies in distribution networks is an unstoppable trend which has a significant positive impact on the progressive power system decarbonization. However, it has to be considered that conventional distribution systems have been designed to operate as passive networks. Therefore, a massive penetration of distributed generation may create several operational problems, such as malfunction of the protection systems, which may limit the deployment of this technology. The analysis of these limitations by means of representative benchmark networks is of utmost importance. Most of the proposed benchmark networks proposed so far, however, lack of information about their protection system. To overcome this shortcoming, this letter specifies the protection system of the benchmark European distribution networks proposed by the CIGRE Task Force C06.04.02 for this purpose. In this way, this letter facilitates the analysis of the possible impact that renewable energy sources may have in the distribution system protections.
\end{abstract}

Keywords Distribution networks · Protection systems · Renewable generation

\section{Introduction}

Traditional low voltage (LV) and medium voltage (MV) distribution systems are planned and operated in a radial manner to provide the energy to passive customers. Taken into account this operation, the power flows from the primary substations to the end users in an unidirectional manner [1]. The protection philosophy of this distribution system is extremely simple, being required just overcurrent protections to detect and isolate short-circuit faults. Circuit breakers, located at the primary substation, and fuses are used for MV and LV grids, respectively, most of the cases. However, this distri-

This research was funded by the Spanish Ministry of Economy and Competitiveness, the European Union Horizon 2020 and the Centre for the Development of Industrial Technology under Grant Numbers ENE2017-84813-R, 764090 and CER-20191019 respectively.

$凶$ J. M. Maza-Ortega

jmmaza@us.es

1 Department of Electrical Engineering, Universidad de Sevilla, Camino de los Descubrimientos s/n, 41092 Seville, Spain

2 Department of Electrical and Computer Engineering, Aristotle University of Thessaloniki, Building D, University Campus, 54124 Thessaloníki, Greece bution paradigm is evolving with the advent of distributed renewable energy sources (DRES) which is key for achieving a progressive decarbonization of the power system generation mix. The impact that a massive DRES penetration may have is usually analyzed by suitable benchmark networks representing real-world cases. Some institutions and working groups have shed light on this issue proposing different distribution benchmark networks. The Test Feeder Working Group of the IEEE PES Distribution System Analysis Subcommittee made publicly available a wide range of distribution test feeders which are summarized and reviewed in $[2,3]$. In addition, EPRI developed two sets of distribution feeder models that are representative of actual small- to large-scale distribution networks [4,5]. The Pacific Northwest National Laboratory introduced a taxonomy of prototypical radial distribution feeder models [6,7] to facilitate the analysis of the upcoming smart grid technologies [8]; the Pacific Gas and Electric Company and the California Energy Commission provided 12 prototypical feeders developed through a cluster analysis [3,9]. The Joint Research Center of the European Commission published a technical report with the most comprehensive data collection of the European distribution systems so far [10]. Similarly, the CIGRE Task Force C6.04.02 [11] presented a comprehensive set of test systems 


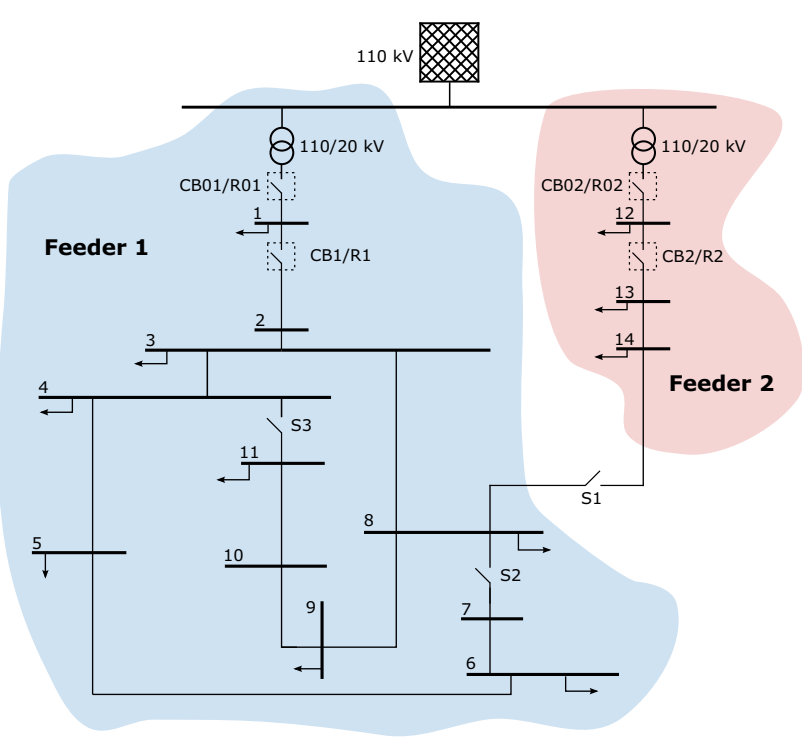

Fig. 1 CIGRE MV distribution network

Table 1 Parameters used for defining the protection settings of the MV distribution system

\begin{tabular}{lllll}
\hline $\begin{array}{l}\text { Circuit } \\
\text { breaker }\end{array}$ & $\begin{array}{l}I_{\mathrm{lth}}, I_{\mathrm{tr}} \\
(\mathrm{A})\end{array}$ & $\begin{array}{l}I_{\mathrm{sc}-3 P}^{\max } \\
(\mathrm{kA})\end{array}$ & $\begin{array}{l}I_{\mathrm{sc}-3 P}^{\min } \\
(\mathrm{kA})\end{array}$ & $\begin{array}{l}I_{\mathrm{sc}-1 P}^{\min } \\
(\mathrm{kA})\end{array}$ \\
\hline $\mathrm{R} 1, \mathrm{R} 01$ & 285,722 & $2.730(\mathrm{~N} 1)$ & $1.089(\mathrm{~N} 7)$ & $839(\mathrm{~N} 7)$ \\
$\mathrm{R} 2, \mathrm{R} 02$ & 276,722 & $2.553(\mathrm{~N} 12)$ & $1.818(\mathrm{~N} 14)$ & $1.244(\mathrm{~N} 14)$ \\
\hline
\end{tabular}

in an attempt to facilitate the analysis and validation of novel techniques and methods, aiming at the DRES integration in an efficient and economic way.

All these benchmark networks allow performing a wide spectrum of studies including major areas such as operation and control [12], planning and design [13], power quality [14], stability issues [15] and short-circuit fault analysis [16]. Most of them, however, lack of any information of their protection system being, therefore, impossible to analyze the impact of DRES penetration on this essential grid component. Note that system protection with a massive DRES integration is a conspicuous issue because these generation units must possess fault ride-through capability. This behavior may interfere the adequate performance of the protection system, usually designed assuming a passive network hypothesis [1], which might pose limits to the DRES hosting capacity of distribution grids.

The aim of this letter is to fill this gap, similarly than [17], by proposing an off-the-shelf overcurrent protection system for the CIGRE Task Force C06.04.02 MV and LV benchmark distribution networks extensively used for the analysis of DRES penetration. In this way, it should be possible to analyze the impact that a massive DRES penetration may have on the actual protection systems of the distribution grid.

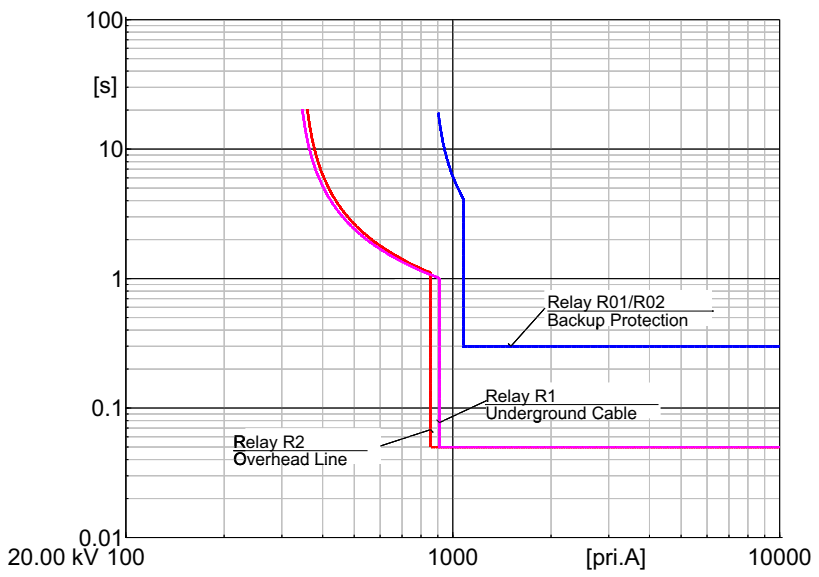

Fig. 2 CIGRE MV network: feeder protection curves

\section{CIGRE TF C06.04.02 MV benchmark network}

\subsection{Network description}

This benchmark system is the reduced version of a MV network in southern Germany and is representative of the typical European MV distribution. As depicted in Fig. 1, it is composed of 14 nodes divided into two $20-\mathrm{kV}$ feeders, where residential, industrial and commercial loads are connected. Three isolation switches (S1, S2 and S3) allow to reconfigure the network topology. All the network details are included in [11].

\subsection{Protection system design}

The MV system is protected through a circuit breaker at each feeder header (R1 and R2) and the corresponding back-up circuit breakers (R01 and R02) in the MV side of the HV/MV transformers as depicted in Fig. 1. The protection system is designed according to the guidelines provided in $[18,19]$ and considering a passive network behavior. For this purpose, the relevant short-circuit currents are calculated using DIgSILENT PowerFactory 2020 SP3 according to the standard IEC60909. Table 1 summarizes the obtained results including in brackets the corresponding node where the short-circuit fault is computed.

The relay settings are summarized in Table 2 according to:

$I_{\mathrm{FSC}}=\max \left[1.5 I_{\mathrm{lth}}, \min \left(0.9 I_{\mathrm{sc}, \min -3 P}, 3.0 I_{\mathrm{lth}}\right)\right]$

$I_{\mathrm{BSC}}=\max \left[1.5 I_{\mathrm{tr}}, \min \left(0.9 I_{\mathrm{sc}, \min -3 P(\text { all })}, 3.0 I_{\mathrm{tr}}\right)\right]$

where $I_{\mathrm{FSC}}$ and $I_{\mathrm{BSC}}$ are the short-circuit settings (ANSI 50) of the main feeder and back-up protection relays, respectively, $I_{\text {th }}$ is the feeder rated thermal current, $I_{\mathrm{sc}, \min -3 P}$ is the 
Table 2 Definition of the protecting devices for the MV distribution system

Fig. 3 LV CIGRE benchmark distribution network

Table 3 Fuses used for protecting the feeders, short-circuit currents and fusing times

\begin{tabular}{|c|c|c|c|c|c|c|}
\hline \multirow[t]{2}{*}{ Circuit breaker } & \multirow{2}{*}{$\begin{array}{l}I_{\mathrm{cs}} \\
(\mathrm{kA})\end{array}$} & \multirow{2}{*}{$\begin{array}{l}\text { ANSI } 51 \\
I_{\mathrm{OC}}(\mathrm{A})\end{array}$} & \multicolumn{2}{|l|}{ ANSI 50} & \multicolumn{2}{|c|}{ ANSI 50N } \\
\hline & & & $\overline{I_{\mathrm{SC}}(\mathrm{A})}$ & Delay (ms) & $\overline{I_{\mathrm{SC}}(\mathrm{A})}$ & Delay $(\mathrm{ms})$ \\
\hline $\mathrm{R} 1$ & 12.5 & 342 & 855 & 50 & 85 & 50 \\
\hline $\mathrm{R} 2$ & 12.5 & 331 & 828 & 50 & 85 & 50 \\
\hline R01, R02 & 12.5 & 866 & 1083 & 300 & 215 & 500 \\
\hline
\end{tabular}

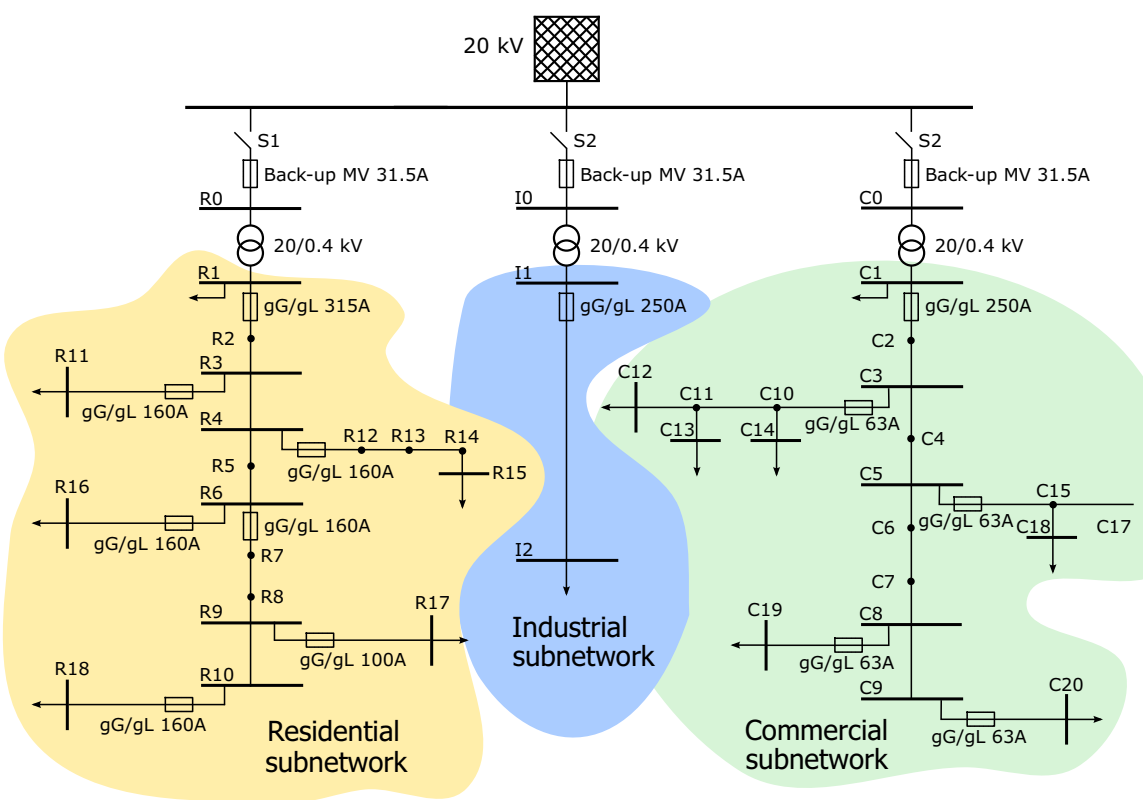

\begin{tabular}{lccccccrc}
\hline Branch & $I_{z}(\mathrm{~A})$ & $I_{n}(\mathrm{~A})$ & $I_{\mathrm{sc}}^{\max }(\mathrm{kA})$ & $t_{c}^{\max }(\mathrm{s})$ & $t_{f}^{\max }(\mathrm{s})$ & $I_{\mathrm{sc}}^{\min }(\mathrm{kA})$ & $t_{c}^{\min }(\mathrm{s})$ & $t_{f}^{\min }(\mathrm{s})$ \\
\hline \multicolumn{2}{l}{ Residential subsystem } & & & & & & & \\
$\mathrm{R} 1-\mathrm{R} 6$ & 435 & 315 & 16.85 & 1.74 & $<0.01$ & 3.48 & 40.05 & 0.66 \\
R6-R10 & 435 & 160 & 5.64 & 15.29 & $<0.01$ & 2.08 & 111.47 & 0.06 \\
$\mathrm{R} 3-\mathrm{R} 11$ & 180 & 160 & 9.72 & 0.22 & $<0.01$ & 3.44 & 1.77 & $<0.01$ \\
R4-R15 & 180 & 160 & 7.86 & 0.10 & $<0.01$ & 1.15 & 17.71 & 0.56 \\
R6-R16 & 180 & 160 & 5.64 & 0.10 & $<0.01$ & 2.29 & 3.98 & 0.04 \\
R9-R17 & 180 & 100 & 3.93 & 0.10 & $<0.01$ & 1.72 & 7.07 & 0.02 \\
R10-R18 & 180 & 100 & 3.57 & 0.10 & $<0.01$ & 1.59 & 8.29 & 0.02 \\
Industrial subsystem & & & & & & & \\
I1-I2 & 330 & 250 & 5.47 & 6.58 & 0.02 & 1.90 & 56.94 & 0.84 \\
Commercial subsystem & & & & & & & \\
C1-C9 & 275 & 224 & 10.57 & 0.32 & $<0.01$ & 1.11 & 29.00 & 3.80 \\
C3-C12 & 88 & 63 & 4.56 & 0.08 & $<0.01$ & 1.06 & 1.64 & 0.01 \\
C5-C17 & 88 & 63 & 2.81 & 0.23 & $<0.01$ & 0.87 & 2.43 & 0.02 \\
C8-C19 & 88 & 63 & 1.77 & 0.57 & $<0.01$ & 0.94 & 2.08 & 0.02 \\
C9-C20 & 88 & 63 & 1.58 & 0.75 & $<0.01$ & 0.86 & 2.51 & 0.02 \\
\hline
\end{tabular}




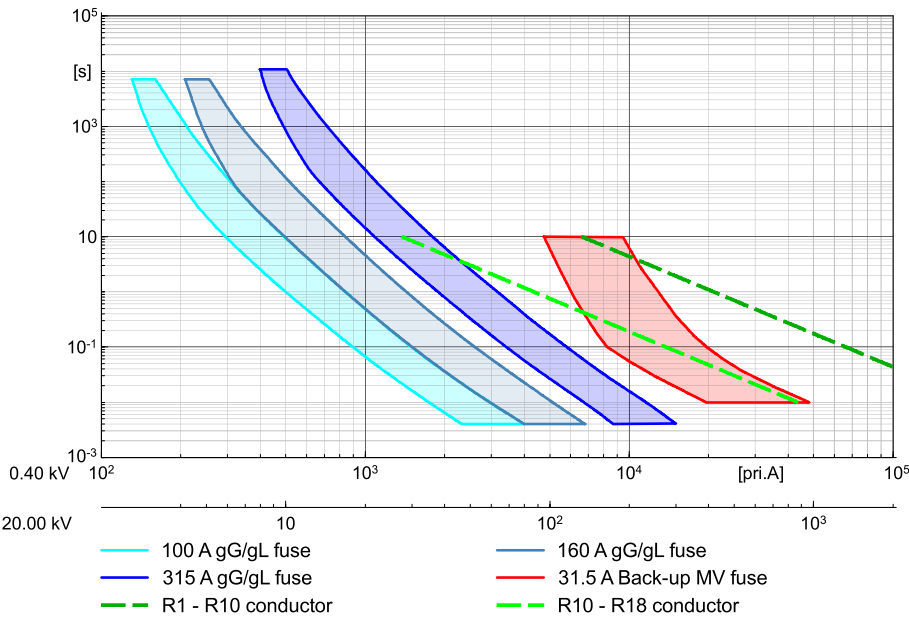

(a)

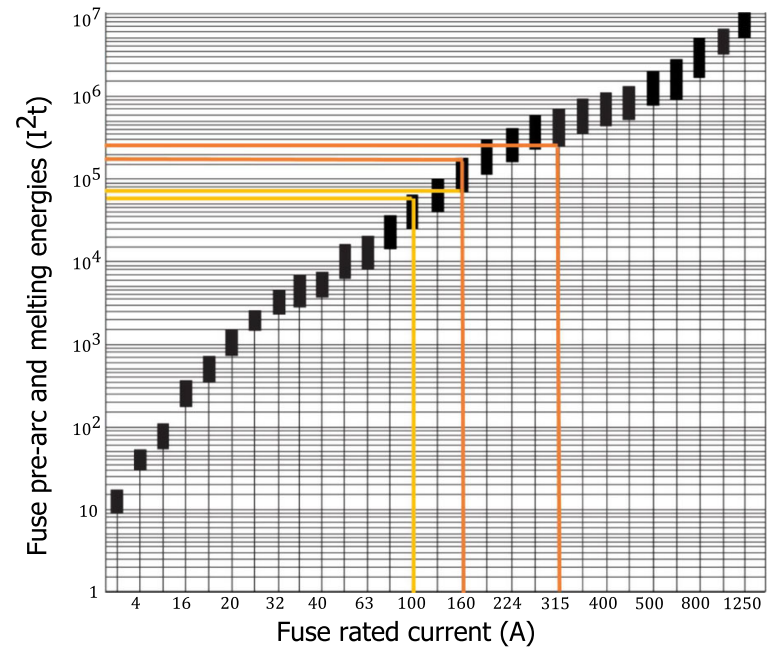

(b)

Fig. 4 Residential feeder path R1-R6-R10-R18: a fuse and cable damage $I-t$ curves; b Pre-arc and melting energy of the selected fuses

feeder minimum short-circuit current, $I_{\mathrm{tr}}$ is the transformer rated current and $I_{\mathrm{sc}, \min -3 P(\text { all })}$ is the minimum short-circuit current of all feeders connected to the transformer. Table 2 also includes the rated short-circuit breaking capacity $\left(I_{\mathrm{cs}}\right)$ which must be higher than the corresponding maximum short-circuit current detailed in Table 1. Time settings of the feeder protection relays are set to $0(50-60 \mathrm{~ms}$ circuit breaker opening time). Conversely, back-up protection is by definition slower than the main feeder protection due to selectivity reasons. Hence, the time settings for the back-up protection are set to $300 \mathrm{~ms}$ in order to ensure graduation between main and back-up protections. The over-current protection (ANSI 51 ) is designed considering that in the case of MV networks the actual loads are unknown. For this reason, the relay pickup currents are set to 1.1-1.2 times the line rated current and transformer rated current for the main feeder and back-up protections, respectively. Figure 2 represents the $I-t$ curves of these relays, where it can be observed the selectivity of the main and back-up protections.

Finally, regarding the instantaneous ground fault protection (ANSI 50N), it has been set to about $30 \%$ of the rated line current and rated transformer current for the main feeder and back-up protections, respectively.

\section{CIGRE TF C06.04.02 LV benchmark network}

\subsection{Network description}

The LV system represents a real three-phase four-wire network with three feeders supplying residential, commercial and industrial loads as shown in Fig. 3. The rated voltage is $400 \mathrm{~V}$, and each subsystem is connected to a $20-\mathrm{kV}$ MV network through different $20 / 0.4 \mathrm{kV}$ transformers. All the network data are available in [11].

\subsection{Protection system design}

The protection system is based on fuses as shown in Fig. 3 complying with [20] and dimensioned according to [21]. Note that the network laterals are protected by their own fuses because the corresponding cables have a lower cross section than the main feeder one. Table 3 contains all the data of the selected fuses with indication of the feeder rated current, $I_{z}$, rated fuse current, $I_{n}$, and the analysis of the protection for the maximum and minimum short-circuit currents. These have been computed using DIgSILENT PowerFactory 2020 SP3 according to the standard IEC60909. Maximum shortcircuit currents, $I_{\mathrm{sc}}^{\max }$, correspond to a three-phase fault at the node where the fuse is installed. Conversely, minimum short-circuit currents, $I_{\mathrm{sc}}^{\mathrm{min}}$, are computed for a single-phase to ground fault at the farthest node of the fuse-protected branch. The melting times for the maximum and minimum short-circuit currents, $t_{f}^{\max }$ and $t_{f}^{\min }$, as well as the cable damage time for these currents, $t_{c}^{\max }$ and $t_{c}^{\min }$, are detailed. Note that an adequate protection is achieved as the melting times are always lower than the cable damage times for both maximum and minimum short-circuit currents.

The MV fuses are detailed in Table 4. However, the backup protection provided by these MV fuses is quite limited because the minimum short-circuit currents within the LV distribution system can be really low. As a matter of fact, the MV fuses are selected to protect the network in case of a three-phase short-circuit fault in the secondary side of the MV/LV transformer. 
Table 4 Back-up MV fuses

\begin{tabular}{ll}
\hline Transformer & $I_{n}(\mathrm{~A})$ \\
\hline Residential subsystem & 31.5 \\
Industrial subsystem & 16 \\
Commercial subsystem & 20 \\
\hline
\end{tabular}

Finally, and in order to evidence the selectivity between the selected fuses, Fig. 4a shows the $I-t$ curves of the fuses installed in the residential subsystem to protect the path comprising the branches R1-R6, R6-R10 and R10-R18. In this case, the length of the main feeder is longer than the critical distance, i.e., the distance where the minimum short-circuit melts the fuse in $5 \mathrm{~s}$. Therefore, the feeder is divided into two sections (R1-R6 and R6-R10) protected by a 315 A and a 160 A fuse, respectively, whereas the lateral (R10$\mathrm{R} 18)$ is protected by a $100 \mathrm{~A}$ fuse. It is important to note that the selectivity is guaranteed if the melting energy $\left(I^{2} t\right)$ of the downstream fuses is lower than the pre-arc energy of the upstream ones. This is represented in Fig. $4 \mathrm{~b}$ where the $I^{2} t$ ranges for the normalized $\mathrm{gG} / \mathrm{gL}$ fuses are shown. The lower and upper values of each bar correspond to the prearc energy and melting energy, respectively. Therefore, and according to Fig. 4, the selected fuses in the analyzed path of the residential sub-network verify the selectivity criterion.

\section{Conclusions}

This letter has defined an off-the-shelf overcurrent protection system for the CIGRE Task Force C06.04.02 European distribution networks which are extensively used for the analysis of DRES penetration. The letter contributes on setting a common base for the researchers dealing with DRES integration to extent their analysis with issues related to the protection systems.

Funding Open Access funding provided thanks to the CRUE-CSIC agreement with Springer Nature.

Open Access This article is licensed under a Creative Commons Attribution 4.0 International License, which permits use, sharing, adaptation, distribution and reproduction in any medium or format, as long as you give appropriate credit to the original author(s) and the source, provide a link to the Creative Commons licence, and indicate if changes were made. The images or other third party material in this article are included in the article's Creative Commons licence, unless indicated otherwise in a credit line to the material. If material is not included in the article's Creative Commons licence and your intended use is not permitted by statutory regulation or exceeds the permitted use, you will need to obtain permission directly from the copyright holder. To view a copy of this licence, visit http://creativecomm ons.org/licenses/by/4.0/.

\section{References}

1. Nimpitiwan N, Heydt GT, Ayyanar R, Suryanarayanan S (2007) Fault current contribution from synchronous machine and inverter based distributed generators. IEEE Trans Power Deliv 22:634-641

2. Schneider KP et al (2018) Analytic considerations and design basis for the IEEE distribution test feeders. IEEE Trans Power Syst 33:3181-3188

3. Marcos FEP et al (2017) A review of power distribution test feeders in the United States and the need for synthetic representative networks. Energies 10:1896

4. Electric Power Research Institute - EPRI (2010) Distributed PV monitoring and feeder analysis. EPRI, Washington, DC

5. Electric Power Research Institute - EPRI (2008) OpenDSS. EPRI test circuits. https://sourceforge.net/p/electricdss/code/HEAD/ tree/trunk/Distrib/EPRITestCircuits/. Accessed 26 Jan 2020

6. Pacific Northwest National Laboratory (2008) GridLAB-D. Taxonomy feeders. http://gridlab-d.shoutwiki.com/wiki/Feeder_ Taxonomy. Accessed 26 Jan 2020

7. Schneider KP, Chen $\mathrm{Y}$ et al (2008) Modern grid initiative: distribution taxonomy final report. https://www.pnnl.gov/ main/publications/external/technical-reports/PNNL-18035.pdf Accessed 26 Jan 2020

8. Schneider KP, Chen Y et al (2009) A taxonomy of North American radial distribution feeders. In: 2009 IEEE PES general meeting. Calgary, Canada

9. Pacific Gas and Electric (PG\&E) (2015) Prototypical feeder models. http://gridlab-d.shoutwiki.com/wiki/PGE_Prototypical_ Models Accessed 26 Jan 2020

10. JRC Technical Reports (2016) Distribution system operators observatory: from European electricity distribution systems to representative distribution networks. https://publications.jrc.ec.europa.eu/ repository/bitstream/JRC101680/ldna27927enn.pdf Accessed 26 Jan 2020

11. Strunz K et al (2014) Benchmark systems for network integration of renewable and distributed energy resources. CIGRE Task Force C6(04):02

12. Martinez-Ramos JL, Zarco-Soto FJ et al (2018) Coordination of distributed energy resources to solve voltage problems in distribution networks. In: 2018 international conference on smart energy systems and technologies (SEST). Sevilla, Spain

13. Zangeneh A, Jadid S, Rahimi-Kian A (2011) A fuzzy environmental-technical-economic model for distributed generation planning. Energy 36:3437-3445

14. Ul-Haq A, Cecati C, Ehsan A, Strunz K (2015) Impact of electric vehicles on voltage profile and harmonics in a distribution network. In: First workshop on smart grid and renewable energy, Doha, Qatar

15. Abdel-Akher M (2013) Voltage stability analysis of unbalanced distribution systems using backward/forward sweep load-flow analysis method with secant predictor. IET Gen Trans Dist 7:309317

16. Plet CA, Graovac M, Green TC, Iravani R (2010) Fault response of grid-connected inverter dominated networks. In: IEEE PES general meeting, Providence, USA

17. Funmilayo JA, Silva JA, Butler-Purry KL (2012) Overcurrent protection for the IEEE 34-node radial test feeder. IEEE Trans Power Deliv 27:459-468

18. ABB S.p.A. Unità Operativa Sace-MV (2016) Technical guide protection criteria for medium voltage networks

19. Siemens AG, Energy Management Medium Voltage Systems (2015) Planning of electric power distribution-technical principles

20. IEC 60269-2 (2013) Low-voltage fuses-part 2: supplementary requirements for fuses for use by authorized persons (fuses mainly 
for industrial application)—examples of standardized systems of fuses $\mathrm{A}$ to $\mathrm{K}$

21. IEC 60364-4-43 (2008) Low-voltage electrical installations-part 4-43: protection for safety-protection against overcurrent
Publisher's Note Springer Nature remains neutral with regard to jurisdictional claims in published maps and institutional affiliations. 